hep-th/9705212

MRI-PHY/970512

\title{
Kaluza-Klein Dyons in String Theory
}

\author{
Ashoke Sentz \\ Mehta Research Institute of Mathematics \\ and Mathematical Physics \\ Chhatnag Road, Jhusi, Allahabad 221506, INDIA
}

\begin{abstract}
S-duality of hetertotic / type II string theory compactified on a six dimensional torus requires the existence of Kaluza-Klein dyons, carrying winding charge. We identify the zero modes of the Kaluza-Klein monopole solution which are responsible for these dyonic excitations, and show that we get the correct degeneracy of dyons as predicted by Sduality. The self-dual harmonic two form on the Euclidean Taub-NUT space plays a crucial role in this construction.
\end{abstract}

\footnotetext{
${ }^{1}$ On leave of absence from Tata Institute of Fundamental Research, Homi Bhabha Road, Bombay 400005, INDIA

${ }^{2}$ E-mail: sen@mri.ernet.in, sen@theory.tifr.res.in
} 
Type II / heterotic string theory compactified on $T^{6}$ has been conjectured to have an exact SL(2,Z) S-duality symmetry that forms a subgroup of the full duality group of these theories [1, 2]. Acting on an elementary string state carrying pure winding charge along one of the compact directions, the S-duality transformation produces a state carrying Kaluza-Klein magnetic charge, as well as winding charge. The existence of these states is a prediction of S-duality symmetry. Furthermore, if S-duality is an exact symmetry of the theory, then these states must have degeneracy identical to that of the elementary string state carrying pure winding charge. In this letter we shall verify this prediction for a class of Kaluza-Klein dyons, namely those with one unit of magnetic charge.[

Let us denote the six internal directions labelling $T^{6}$ by $x^{4}, \ldots x^{9}$. The momentum and winding charge quantum numbers along $x^{4}$ are labelled by a 2 dimensional vector:

$$
\vec{\alpha}=\left(\begin{array}{c}
p \\
w
\end{array}\right),
$$

where $p$ and $w$ are integers. Suppose $n_{K}$ and $n_{H}$ are integers labelling Kaluza-Klein monopole charge and $H$-monopole charge associated with the $x^{4}$ direction respectively. Then we can define another two dimensional vector $\vec{\beta}$ through the relation [1]

$$
L \vec{\beta}=\left(\begin{array}{l}
n_{K} \\
n_{H}
\end{array}\right),
$$

where

$$
L=\left(\begin{array}{ll}
0 & 1 \\
1 & 0
\end{array}\right),
$$

is the natural metric on the two dimensional lattice of momentum and winding charges along $x^{4}$. SL $(2, \mathrm{Z})$ S-duality group acts on $(\vec{\alpha}, \vec{\beta})$ as $\mathbb{1}$

$$
\left(\begin{array}{l}
\vec{\alpha} \\
\vec{\beta}
\end{array}\right) \rightarrow\left(\begin{array}{ll}
l & q \\
r & s
\end{array}\right)\left(\begin{array}{l}
\vec{\alpha} \\
\vec{\beta}
\end{array}\right),
$$

where

$$
l, q, r, s \in Z, \quad l s-q r=1 .
$$

An elementary string state carrying one unit of winding charge along $x^{4}$ has

$$
\vec{\alpha}=\left(\begin{array}{l}
0 \\
1
\end{array}\right), \quad \vec{\beta}=0 .
$$

\footnotetext{
${ }^{3}$ Classical solutions carrying Kaluza-Klein magnetic charge and winding charge can be constructed by using the standard trick of duality rotation[3] (see, for example, 《]).
} 
Acting on this state, the $\mathrm{SL}(2, \mathrm{Z})$ transformation given in eq.(任) produces a state with

$$
\vec{\alpha}=\left(\begin{array}{l}
0 \\
l
\end{array}\right), \quad \vec{\beta}=\left(\begin{array}{l}
0 \\
r
\end{array}\right),
$$

i.e.

$$
w=l, \quad n_{K}=r .
$$

Standard argument shows that as a consequence of eq.(5), $l$ and $r$ are relatively prime. Thus according to S-duality, for every pair of integers $l$ and $r$ relatively prime, the theory must contain dyons with $r$ units of Kaluza-Klein magnetic charge and $l$ units of winding charge. The degeneracy of these states must match the degeneracy of the elementary string states carrying quantum numbers (6).

We shall focus on states with $r=1$ and identify the dyonic excitations of the monopole carrying winding charge.t We begin by writing down the Kaluza-Klein monopole solution in ten dimensional string metric[6, 7]

$$
d s^{2}=-d t^{2}+\sum_{m=5}^{9} d x^{m} d x^{m}+d s_{T N}^{2},
$$

where $d s_{T N}$ denotes the Euclidean Taub-NUT metric:

$$
\begin{gathered}
d s_{T N}^{2}=V\left\{d x^{4}+4 m(1-\cos \theta) d \phi\right\}^{2}+V^{-1}\left(d r^{2}+r^{2} d \theta^{2}+r^{2} \sin ^{2} \theta d \phi^{2}\right), \\
V=\left(1+\frac{4 m}{r}\right)^{-1} .
\end{gathered}
$$

In order for the solution to be non-singular at the origin, we need the periodicity of $x^{4}$ to be $16 \pi m$.

This solution has three bosonic zero modes, which are simply the translational modes of the solution in the Euclidean space labelled by the polar coordinates $(r, \theta, \phi)$. The momenta conjugate to these zero modes represent spatial momenta of the monopole. Note that since the solution is invariant under a translation along the $x^{4}, \ldots x^{9}$ direction, there is no zero mode associated with translation in these directions, and hence the monopole cannot carry momentum along these directions.

If these were all the bosonic deformations of the solution, there would not be any dyonic excitation of the solution carrying winding charge along the $x^{4}$ direction. However, the presence of the anti-symmetric tensor gauge field $B_{\mu \nu}$ in this theory, and the existence of a

\footnotetext{
${ }^{4}$ These are $T$-dual to the $H$-dyon states analysed in ref. $[5]$.
} 
harmonic two form in the Euclidean Taub-NUT space[8, 9, 10, 11], allows us to construct new bosonic zero modes of the solution, by considering deformations of the form:

$$
B=\Theta \Omega,
$$

where $\Theta$ is the deformation parameter, and $\Omega$ is the self-dual harmonic two form:

$$
\Omega=C \frac{r}{r+4 m}\left(d \sigma_{3}+\frac{4 m}{r(r+4 m)} d r \wedge \sigma_{3}\right) .
$$

Here $C$ is a normalization constant, and,

$$
\sigma_{3}=(4 m)^{-1}\left(d x^{4}+4 m(1-\cos \theta) d \phi\right) .
$$

The normalization constant $C$ is chosen in such a way that the imaginary part of the string action for an Euclidean world sheet, wrapped on a (non-compact) two cycle dual to $\Omega$, is given by $i \Theta$. This will make the coordinate $\Theta$ periodic with period $2 \pi$.

Note that $\Omega$ can be written as,

$$
\Omega=d \xi
$$

where

$$
\xi=C \frac{r}{r+4 m} \sigma_{3} .
$$

$\xi$ represents a non-singular one form at $r=0$, but does not vanish as $r \rightarrow \infty$. This shows that the zero mode deformation that we have introduced corresponds to a pure gauge deformation of the solution, with the gauge transformation parameter not vanishing at $\infty$. The situation is exactly analogous to the case of BPS monopoles, for which the collective coordinate conjugate to the electric charge corresponds to a pure gauge deformation. Since $\Theta \rightarrow \Theta+\alpha$ is a gauge transformation, the collective Hamiltonian does not depend explicitly on $\Theta$. Hence the momentum $p_{\Theta}$ conjugate to $\Theta$ is conserved, and can be interpreted as the winding charge. Up Upon quantization $p_{\Theta}$ is quantized in integer units, and would correspond to the winding number $w$.

\footnotetext{
${ }^{5}$ For type II theories, one can get other deformations involving Ramond-Ramond gauge fields. These will be responsible for constructing dyonic states carrying Ramond-Ramond charge, as predicted by Uduality, but we shall not consider them here. Also, one needs to take into account these deformations to get the correct counting of zero modes for Kaluza-Klein monopoles, as given in [12].

6 To see this let us consider a configuration of constant $p_{\Theta}$, for which $\Theta=\alpha t+\Theta_{0}$. Using eqs.(12)-(14) we see that for this solution, as $r \rightarrow \infty$,

$$
H=d B \simeq \alpha C r^{-2} d t \wedge d r \wedge\left(d x^{4}+4 m(1-\cos \theta) d \phi\right)+\alpha C \sin \theta d t \wedge d \theta \wedge d \phi
$$

The term in $H$ proportional to $d t \wedge d r \wedge d x^{4}$ indicates that the solution carries winding charge $\propto \alpha \propto p_{\Theta}$. The other terms can be interpreted as non-trivial axion background in the four dimensional theory.
} 
This establishes the existence of the required dyonic excitations carrying $n_{K}=1$, $w$ arbitrary. In order to verify the predictions of S-duality, we also need to make sure that these states have degeneracy identical to that of a singly wound elementary BPS excitation in the corresponding string theory. For type II string theory, the degeneracy of the elementary string state is 256, corresponding to an ultra-short multiplet of the supersymmetry algebra. For the Kaluza-Klein dyons, this degeneracy comes from quantizing the fermionic zero modes associated with the broken supersymmetry generators. Since the monopole solution breaks half of the thirty two supersymmetry generators, the sixteen broken generators give rise to 16 fermionic zero modes, whose quantization gives a $2^{8}=256$-fold degenerate state. Thus for type II theory on $T^{6}$, we get exactly the right degeneracy for the Kaluza-Klein dyons.

The situation in the heterotic string theory is somewhat more complicated. In this case, in the elementary string spectrum, a BPS state carrying pure winding charge is $16 \times$ 24 fold degenerate, corresponding to 24 short multiplets. (The number 24 is related to the number of bosonic oscillators in the left-moving sector of the theory.) On the other hand, the Kaluza-Klein monopole solution breaks eight of the sixteen supersymmetry generators. This gives 8 fermionic zero modes, and hence $2^{4}=16$-fold degeneracy, corresponding to one short multiplet. Thus we are missing a factor of 24 .

The resolution to this problem comes from the fact that the Kaluza-Klein monopole solution that we have displayed carries one unit of gravitational instanton number [13], and hence acts as a source of -1 unit of $H$-magnetic charge. These states are dual to elementary string states with $(p=-1, w=1)$, which indeed have degeneracy 16 , corresponding to a single short multiplet. If we want a state whose magnetic charge is only of the Kaluza-Klein type, we must cancel this $H$-magnetic charge. This can be done by placing a gauge instanton inside the Euclidean Taub-NUT space. At a generic point in the moduli space of the theory, the gauge group is purely abelian, and hence the instanton must necessarily have zero size. This corresponds to a heterotic five-brane wrapped on the $T^{5}$ labelled by $x^{5}, \ldots x^{9}$. This will have extra fermionic and bosonic zero modes which must be taken into account in computing the degeneracy of the state. However, this is precisely the problem that has been addressed in the context of calculating degeneracy of H-monopoles [14, 15], and it is known that the quantization of these degrees of freedom enhances the degeneracy by a factor of 24 16, 17, 18]. In the present case we also get a set of extra bosonic and fermionic zero modes associated with the location of the small 
instanton in the Taub-NUT space and their fermionic partners. Quantization of these zero modes gives an extra multiplicative factor in the degeneracy equal to the number of normalizable harmonic forms on the Taub-NUT space. This number is known to be unity [10]. This then gives the right counting of the degeneracy of states of Kaluza-Klein dyons for the heterotic string theory.

Acknowledgement: I wish to thank C. Hull and E. Witten for useful correspondence.

\section{References}

[1] A. Sen, Int. J. Mod. Phys. A9 (1994) 3707 hep-th/9402002 and references therein.

[2] C. Hull and P. Townsend, Nucl. Phys. B438 (1995) 109 hep-th/9410167.

[3] A. Shapere, S. Trivedi and F. Wilczek, Mod. Phys. Lett. A6 (1991) 2677.

[4] M. Cvetic and D. Youm, Phys. Rev.D53 (1996) 584 hep-th/9507090.

[5] J. Blum, hep-th/9702084.

[6] R. Sorkin, Phys. Rev. Lett. 51 (1983) 87.

[7] D. Gross and M. Perry, Nucl. Phys. B226 (1983) 29.

[8] D. Brill, Phys. Rev. B133 (1964) 845;

C. Pope, Nucl. Phys. B141 (1978) 432.

[9] J. Gauntlett and D. Lowe, Nucl. Phys. B472 (1996) 194 hep-th/9601085].

[10] K. Lee, E. Weinberg and P. Yi, Phys. Lett. B376 (1996) 97 hep-th/9601097.

[11] S. Connell, Univ. of South Australia preprint.

[12] C. Hull, hep-th/9705162.

[13] S. Hawking, Phys. Lett. 60A (1977) 81;

G. Gibbons and S. Hawking, Comm. Math. Phys. 66 (1979) 291.

[14] T. Banks, M. Dine, H. Dijkstra and W. Fischler, Phys. Lett. B212 (1988) 45.

[15] J. Gauntlett and J. Harvey, hep-th/9407111. 
[16] E. Witten, Nucl. Phys. B460 (1996) 541 [hep-th/9511030].

[17] M. Porrati, Phys. Lett. B387 (1996) 492 hep-th/9607082.

[18] S. Sethi and M. Stern, Phys. Lett. B398 (1997) 47 hep-th/9607145. 